\title{
Madelung disease in a 58-year-old man
}

\author{
Shigehiro Karashima PhD, Takashi Yoneda PhD
}

Cite as: CMAJ 2019 January 14;191:E48. doi: 10.1503/cmaj.180759

A 58-year-old man presented to our hospital for surgical resection of a swelling of the upper back (Figure 1). The patient was a heavy drinker who had been given a diagnosis of alcoholic fatty liver at age 35 years. He had no history of glucocorticoid or antiretroviral drug use and no family history of similar swellings. He had a body mass index of 19.0 (normal 18.5-25.0). He did not have moon facies, thin skin or central obesity, and his physical examination was otherwise unremarkable. Laboratory testing showed only minor elevation of hepatic enzymes. His basal cortisol level was normal, as were his $1 \mathrm{mg}$ and $8 \mathrm{mg}$ dexamethasone suppression tests. Computed tomography images showed diffuse, nonencapsulated fatty deposits in the subcutaneous compartments of the supraclavicular fossa and upper back (Appendix 1, available at www.cmaj. ca/lookup/suppl/doi:10.1503/cmaj.180759/-/DC1). We diagnosed Madelung disease, an uncommon condition characterized by a symmetric pattern of massive fatty deposits in the head, neck, trunk and nerve roots of the upper and lower limbs.

The differential diagnosis for Madelung disease includes obesity, Cushing syndrome, effects of medication (for example, antiretroviral therapy for HIV/AIDS or glucocorticoids) and lipid storage disorders. Madelung disease is strongly associated with alcohol consumption. When our patient reduced his average ethanol intake from 13 to 4 standard drinks per day, his fat mass shrank substantially over 6 months of follow-up (Appendix 1). Madelung disease is often managed with lipectomy or liposuction but, as in our case, alcohol avoidance may decrease the fat accumulation. ${ }^{1}$ The cause of Madelung disease is still unknown, but defects in lipolysis, sympathetic denervation of brown fat adipocytes and a mutation of mitochondrial DNA have been put forward as plausible explanations. ${ }^{2-4}$

\section{References}

1. Chen C-Y, Fang Q-Q, Wang X-F, et al. Madelung's disease: Lipectomy or liposuction? Biomed Res Int 2018;2018:3975974.

2. González-García R, Rodríguez-Campo FJ, Sastre-Pérez J, et al. Benign symmetric lipomatosis (Madelung's disease): case reports and current management. Aesthetic Plast Surg 2004;28:108-12.

3. Enzi G, Busetto L, Sergi G, et al. Multiple symmetric lipomatosis: a rare disease and its possible links to brown adipose tissue. Nutr Metab Cardiovasc Dis 2015;25:347-53.

4. Mancuso M, Orsucci D, Angelini C, et al. Phenotypic heterogeneity of the $8344 \mathrm{~A}>\mathrm{G}$ mtDNA “MERRF” mutation. Neurology 2013;80:2049-54.

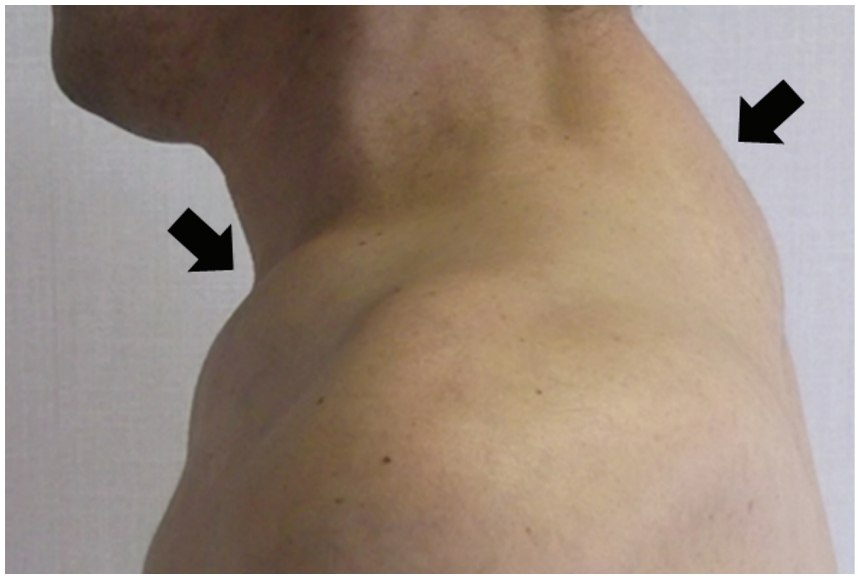

Figure 1: Swelling of the supraclavicular fossa and upper back (arrows) in a 58-year-old man with Madelung disease.

Competing interests: None declared.

This article has been peer reviewed.

The authors have obtained patient consent.

Affiliations: Division of Endocrine and Diabetes (Karashima), Department of Cardiovascular and Internal Medicine, Kanazawa University Graduate School of Medical Sciences; Internal Medicine (Karashima), Ishikawa Prefecture Saiseikai Kanazawa Hospital; Program Management Office for New Paradigms - Establishing Centers for Fostering Medical Researchers of the Future and Institute of Liberal Arts and Science (Yoneda), Kanazawa University, Ishikawa, Japan

Correspondence to: Shigehiro Karashima, skarashima@staff.kanazawa-u.ac.jp

Clinical images are chosen because they are particularly intriguing, classic or dramatic. Submissions of clear, appropriately labelled highresolution images must be accompanied by a figure caption. A brief explanation (300 words maximum) of the educational importance of the images with minimal references is required. The patient's written consent for publication must be obtained before submission. 